\title{
Conhecimento dos profissionais de saúde sobre doenças infectoparasitárias relacionadas à degradação ambiental urbana
}

A urbanização de doenças infectoparasitárias evitáveis a partir de medidas preventivas simples mantém-se constantes nos últimos anos no país. Portanto objetivo deste estudo é identificar o conhecimento dos profissionais de saúde sobre as doenças infectoparasitárias relacionadas à degradação ambiental urbana. Trata-se de uma pesquisa descritiva de abordagem qualitativa, realizada com 39 profissionais das 10 Equipes de Saúde da Família (ESF) localizados na área urbana na cidade de Cáceres/MT, no ano de 2015. A pesquisa foi realizada por meio de questionário fechado sobre transmissão das doenças infectoparasitárias por água contaminada, solo contaminado e áreas precárias de urbanização. A variável água contaminada foi mais bem percebida como problema para doenças infectoparasitárias nos territórios dos ESF estudados. As equipes de saúde da Cohab Nova, Vila Irene e Jardim Paraíso mostraram melhor percepção sobre a variável. No entanto, as equipes apresentam pouco conhecimento sobre as doenças transmitidas/obtidas por solo contaminado e em áreas precárias de urbanização. Conclui-se que as doenças infectoparasitárias são consideradas problemas de saúde ambiental nos territórios das ESF, mas as equipes apresentaram dificuldades em responder os questionários, mostrando possível falta de conhecimento sobre o tema, fato que pode influenciar na saúde da população residente nos territórios estudados. Portanto, torna-se necessário a implantação de educação continuada com as equipes de saúde estudadas.

\section{Knowledge of health professionals on infectoparasitary diseases related to urban environmental degradation}

\begin{abstract}
The urbanization of preventable infectious diseases based on simple preventive measures has remained constant in recent years in the country. Therefore, the objective of this study is to identify the knowledge of health professionals about infectious diseases related to urban environmental degradation. This is a qualitative descriptive research carried out with 39 professionals from the 10 Family Health Teams (ESF) located in the urban area of the city of Cáceres/MT, in the year 2015. The research was carried out through a closed questionnaire on the transmission of infectious diseases by contaminated water, contaminated soil and precarious areas of urbanization. The variable contaminated water was better perceived as a problem for infectious diseases in the territories of the studied ESF. The health teams of Cohab Nova, Vila Irene and Jardim Paraíso showed a better perception about the variable. However, the teams have little knowledge about the diseases transmitted/obtained by contaminated soil and in precarious areas of urbanization. It was concluded that infectious diseases are considered environmental health problems in the territories of the ESF, but the teams presented difficulties in answering the questionnaires, showing a possible lack of knowledge about the subject, a fact that may influence the health of the resident population in the territories studied. Therefore, it is necessary to implement continuing education with the health teams studied.
\end{abstract}

Keywords: Health Team; Urbanization; Infectious Diseases; Parasitic Diseases; Environment.

Topic: Epidemiologia e Saúde Ambiental

Reviewed anonymously in the process of blind peer.
Received: $12 / 02 / 2019$

Approved: 28/03/2019
Danyella Rodrigues de Almeida (iD)

Universidade do Estado do Mato Grosso, Brasil

http://lattes.cnpq.br/6285168230103784

http://orcid.org/0000-0003-1181-9321

dralmeida.unemat@gmail.com

\section{Antonio Francisco Malheiros (iD}

Universidade do Estado do Mato Grosso, Brasil

http://lattes.cnpq.br/9067970026570376

http://orcid.org/0000-0001-8169-0557

malheiros@unemat.br

\section{Aumeri Carlos Bampi}

Universidade do Estado do Mato Grosso, Brasil

http://lattes.cnpq.br/4800812434410023

http://orcid.org/0000-0002-3410-9376

aumeribampi@gmail.com
Referencing this:

ALMEIDA, D. R.; MALHEIROS, A. F.; BAMPI, A. C.. Conhecimento dos profissionais de saúde sobre doenças infectoparasitárias relacionadas à degradação ambiental urbana. Revista Ibero Americana de Ciências Ambientais, v.10, n.2, p.147-154, 2019. DOI:

http://doi.org/10.6008/CBPC2179-6858.2019.002.0013 


\section{INTRODUÇÃO}

O crescimento urbano desordenado em decorrência do fracionamento irregular do solo gera diversos problemas urbanos e ambientais. A precariedade de infraestruturas básicas nas periferias das cidades constituem ameaças à saúde da população (ANDREAZZI et al., 2007), uma vez que o poder público investe pouco em serviços e os moradores convivem com falta de água potável, lixo nas ruas, valas com esgoto a céu aberto, vetores transmissores de doenças e riscos de enchentes. Nesse contexto, a população carente, sem condições e perspectiva de mudanças locais, torna-se agressora do ambiente e receptora principal das consequências da degradação, retroalimentando a pobreza e a degradação ambiental (BARBOSA et al., 2015), aumentado o risco de doenças evitáveis.

As doenças infectoparasitárias fazem parte do cotidiano das famílias brasileiras (PIUVEZAM et al., 2015). Apesar da mortalidade por doenças infectoparasitárias ter reduzido nos últimos anos, verifica-se que essas enfermidades ainda ocupam papel importante na morbimortalidade brasileira. No ano de 2015 foram mais de 55 mil óbitos e 809 mil internações por doenças infectoparasitárias no Brasil (MINISTÉRIO DA SAÚDE, 2016). Vários fatores contribuem com a manutenção e aumento das doenças infectoparasitárias, entre eles fatores demográficos, sociais, políticos, econômicos, ambientais (PIGNATTI, 2004; SCHRAMM et al., 2004) e desempenho do setor saúde.

A urbanização de doenças infectoparasitárias é objeto de preocupação na saúde pública; uma vez que podem ser evitadas com medidas preventivas simples, a partir de ações de educação e promoção da saúde por meio dos trabalhos realizados pelas Equipes de Saúde da Família (ESF), mas anualmente milhares de casos são diagnosticados no país. Portanto, o presente estudo teve como objetivo identificar o conhecimento dos profissionais de saúde sobre as doenças infectoparasitárias relacionadas à degradação ambiental urbana.

\section{METODOLOGIA}

\section{Desenho do estudo}

Estudo qualitativo, com registros das percepções dos profissionais de saúde das ESF de Cáceres/MT acerca do conhecimento das doenças infecciosas e parasitárias relacionadas à degradação ambiental urbana e a relação com a saúde humana.

\section{População e Área do estudo}

A população estudada foi composta por 39 profissionais da área de saúde lotados nos ESF da zona urbana de Cáceres (figura 1), sendo 17 Agentes Comunitários de Saúde (ACS), oito técnicos de enfermagem, nove enfermeiros e cinco médicos. No entanto, o quadro de saúde das ESF é composto por 62 profissionais. O município de Cáceres (figura 2) está localizado na mesorregião Centro-Sul Mato-Grossense, na microrregião do alto Pantanal, a margem esquerda do rio Paraguai, bacia do Alto Paraguai. O município ocupa área territorial de $24.593,031 \mathrm{~km}^{2}$, com população estimada em 90.518 habitantes durante o período 
estudado. Cáceres apresenta 59.6\% de domicílios com esgotamento sanitário adequado, 79.2\% de domicílios urbanos em vias públicas com arborização e $23.2 \%$ de domicílios urbanos em vias públicas com urbanização adequada, com presença de bueiro, calçada, pavimentação e meio-fio (IBGE, 2015). No município há 10 Unidades de Saúde da Família (USF) na área urbana e três na rural. O estudo foi realizado com as equipes das unidades da área urbana (figura 1).

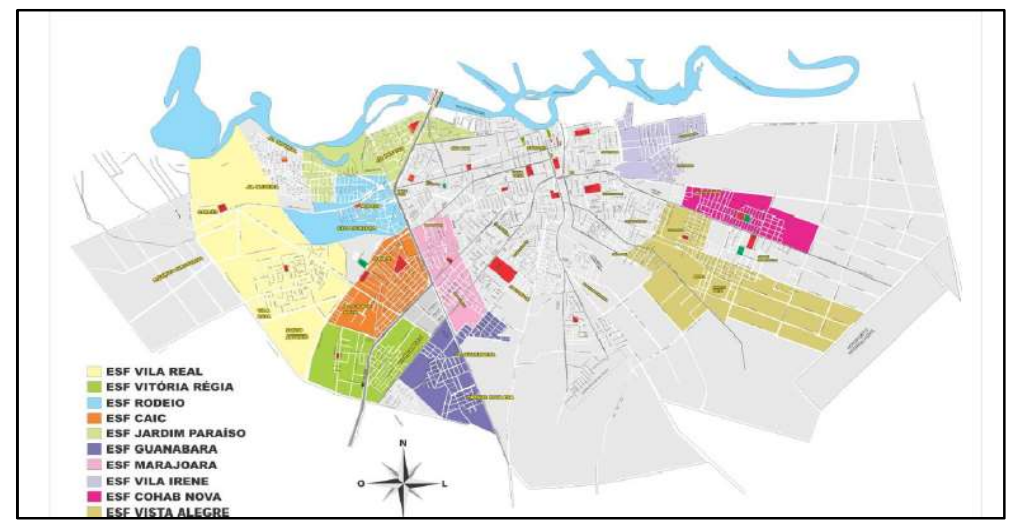

Figura 1: Localização das dez unidades de Estratégias Saúde da Família na área urbana de Cáceres/MT.

\section{Coleta de dados}

O questionário aplicado no estudo foi adaptado de Lima (2013), foram quatro perguntas para estudar a percepção dos profissionais de saúde sobre doenças infectoparasitárias, degradação ambiental urbana e a relação com a saúde humana. As variáveis foram água contaminada, solo contaminado, áreas precárias de urbanização e meio de comunicação para a população. Os dados foram classificados com base na Classificação Ambiental das Doenças Relacionadas ao Saneamento Ambiental Inadequado (DRSAl) (CAIRNCROSS et al., 1993) e doenças relacionadas com a drenagem urbana, por meio da Classificação Ambiental (SOUZA et al., 2002).

Os dados foram coletados entre julho e setembro de 2015. Primeiro, foi realizado uma abordagem da proposta da pesquisa no Centro de Referência de Saúde com todos os enfermeiros das ESF de Cáceres/MT. Posteriormente, foi realizada visita nas unidades de saúde para aplicação do questionário. A coleta de dados foi realizada nas unidades de saúde, foram entregue o Termo de Consentimento Livre e Esclarecido (TCLE) e o questionário com perguntas fechadas com respostas alternativas.

Durante o preenchimento dos questionários, o pesquisador esteve disponível em tempo integral para eventuais dúvidas, no final, todos os questionários não identificados foram recolhidos e colocados em envelope lacrados para manter o sigilo dos dados obtidos. Após a coleta, os dados foram organizados em planilhas eletrônicas e posteriormente foi realizada a análise estatística descritiva da frequência relativa. 


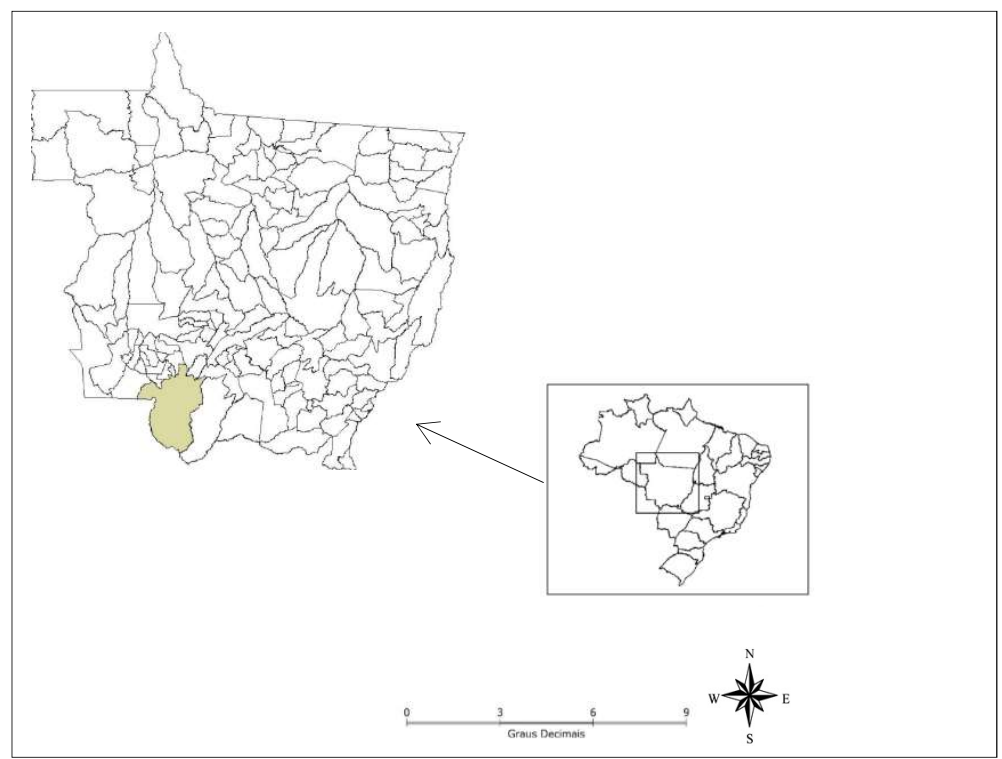

Figura 2: Municípios de Cáceres, estado de Mato Grosso, Brasil.

\section{RESULTADOS}

Foi realizado na cidade de Cáceres, no ano de 2015 um questionário com os profissionais das ESF sobre doenças infecciosas e parasitárias transmitidas e/ou obtidas por água, solo contaminado e áreas precárias de urbanização. A classificação ambiental das doenças relacionadas com a água foi categorizada em feco-oral, higiene, transmissão por vetor, solo contaminado foi categorizada em excretas humanas e lixo e, áreas precárias de urbanização foram consideradas doenças relacionadas com drenagem urbana, excretas humanas e água.

Em relação às doenças transmitidas por água contaminada via feco-oral foi à categoria mais citada pela equipe de saúde, principalmente diarreias e disenterias. As equipes mostraram pouco conhecimento sobre as doenças relacionadas à higiene e as áreas precárias de urbanização. As equipes de saúde da Cohab Nova, Vila Irene e Jardim Paraíso apresentaram maior conhecimento sobre as doenças infecciosas e parasitárias transmitidas por água contaminada. As equipes locadas nos bairros Guanabara, Rodeio e Vitória Régia apresentaram dificuldades para assimilar as doenças com a variável água contaminada (tabela 1).

Em relação às doenças transmitidas por solo contaminado a categoria relacionada com excretas humanas foi a mais citada pelas equipes de saúde. A equipe do Jardim Paraíso apresentou melhor conhecimento sobre o tema, as equipes dos ESF localizadas na Vila Real, Guanabara, Rodeio e Vitória Régia demonstraram não ter nenhum conhecimento sobre as doenças relacionadas com o solo contaminado. Algumas respostas foram desconsideradas por não fazerem parte da classificação ambiental das infecções relacionadas à excretas e lixos, mostrando o desconhecimento das equipes estudadas (tabela 2).

Em relação às doenças presentes em áreas precárias de urbanização a categoria drenagem urbana foi a mais citada pelas equipes de saúde. A equipe da Vista Alegre apresentou melhor conhecimento sobre o tema, as equipes dos ESF localizadas na Cohab Nova, Guanabara, Rodeio e Vitória Régia demonstraram não ter nenhum conhecimento sobre as doenças presentes em áreas precárias de urbanização (tabela 3). 


\section{DISCUSSÃO}

A variável água contaminada foi mais bem percebida como problema para doenças infectoparasitárias nos territórios dos ESF estudados. As equipes de saúde da Cohab Nova, Vila Irene e Jardim Paraíso mostraram melhor percepção sobre a variável contaminação da água. No entanto, as equipes apresentam pouco conhecimento sobre as doenças transmitidas/obtidas em áreas precárias de urbanização, apenas $15,7 \%$ das equipes citaram doenças, 4,3\% não especificaram os agentes etiológicos, 30,0\% das doenças foram desconsideradas e 50,0\% da equipe de saúde não responderam o questionário. Trabalhos educação continuada devem ser realizados com as equipes de saúde de Cáceres, uma vez que o trabalho das equipes multiprofissionais na ESF é um importante instrumento de intervenção.

Diversos fatores estão relacionados com o aumento do risco para doenças infectoparasitárias, entre eles o aumento da população e a falta de planejamento adequado causam problemas no abastecimento de água, esgotamento sanitário e ocupações irregulares. Cairncross et al. (1993) propuseram a classificação para DRSAI. A classificação das DRSAI divide-se em: (i) doenças de transmissão feco-oral; (ii) doenças transmitidas por inseto vetor; (iii) doenças transmitidas pelo contato com a água; (iv) doenças relacionadas com a higiene; e (v) geo-helmintos e teníases.

A água é considerada bem público indispensável para a vida, mas um bilhão de pessoas no mundo não têm acesso à água tratada, destas 19 milhões residem no Brasil (RHEINGANS et al., 2006). Segundo IBGE, 2015, o esgotamento sanitário de Cáceres está adequado em 59,6\% da cidade. No entanto, o restante do esgoto e dejetos é lançado diretamente nos canais fluviais Junco, Sangradouro, José Bastos, Renato, galerias pluviais do perímetro urbano e rio Paraguai (SILVA et al., 2012), os córregos cortam a cidade e apresentam áreas degradadas, presença de lixo, despejo de esgoto, tornando a água de coloração azul esverdeada e odor fétido. Estudos mostram que os pontos Baía do late, Baía do Malheiros e Captação de Água no rio Paraguai apresentam sinais de degradação, erosão e desmatamento por ações antrópicas associadas às atividades comerciais, habitações e atividades recreativas. O recurso hídrico está prejudicado no ponto da Captação de Água e na Baía do Malheiros devido o lançamento de esgoto comercial e residencial sem tratamento no rio. O ponto de Captação de água que abastece a cidade de Cáceres encontra-se em local jusante de onde são despejados dejetos e está a poucos metros da Baía do Malheiros onde há lançamento de esgoto do córrego Sangradouro de forma negligenciada pelas autoridades públicas da cidade (BÜHLER, 2011; BÜHLER et al., 2012; SANTANA et al., 2014). No entanto, há o sistema de tratamento da água antes do abastecimento para população.

Estima-se que 33\% da carga global de doença são atribuíveis a fatores ambientais (PRUSS-USTUN et al., 2008a). O acesso à rede de abastecimento de água e esgoto adequado tem grande impacto na saúde das populações. Investimentos na melhoria do abastecimento de água, saneamento e higiene podem prevenir uma série de agravos, principalmente a diarreia (PRUSS-USTUN et al., 2008b), considerada uma das principais causas de morbimortalidade em países em desenvolvimento (PRUSS-USTUN et al., 2008c; EJEMOT et al., 2012). Estudo mostra que na Região Centro Oeste as doenças infectoparasitárias foram responsáveis por 
7,43\% das internações, 437,72 casos por 100 mil habitantes no ano de 2013, as doenças relacionadas à poluição hídrica foram responsáveis por 2,63\% das internações, 155,12 casos por 100 mil habitantes (PAIVA et al., 2018).

A falta de saneamento acarreta diversos impactos negativos sobre a saúde da população, causando grave ameaça à saúde humana. O saneamento ineficaz pode acarretar doenças veiculadas pela água e propiciar a reprodução de vetores de outras doenças. A população de baixa renda é mais vulnerável a essas doenças, devido à higiene inadequada e à subnutrição (OPS, 2011). As doenças infectoparasitárias transmitidas/obtidas por solo contaminado estão relacionadas com falta de saneamento básico, principalmente pela falta de coleta e disposição inadequada do lixo (RIBEIRO et al.; 2010; LIMA, 2013). Atualmente a cidade de Cáceres conta com um aterro sanitário, desde julho de 2016, mas matérias em sites locais mostram que o aterro não está funcionando como indica a lei 12.305/2010 que instituiu a Política Nacional de Resíduos Sólidos (PNRS) (BRASIL, 2012). Antes do aterro sanitário a área do Lixão estava localizada a 15 quilômetros do perímetro urbano, na Bacia do córrego Piraputangas, cabeceira do Pantanal mato-grossense e cerca de 40 toneladas de lixos comerciais, residenciais e hospitalares (FERREIRA et al., 2017) eram despejados diariamente no local (ALCÂNTARA et al., 2010).

Estudo mostra que doenças relacionadas ao saneamento básico inadequado no Brasil foram responsáveis, em média, por 13.449 óbitos e 758.750 internações hospitalares por ano durante o período de 2001 a 2009. Apesar da acentuada queda da mortalidade as doenças infectoparasitárias ainda constituem a terceira causa de internação hospitalar no país (PONTES et al., 2009).

A cobertura territorial realizada pela ESF pode contribuir para redução dos casos de doenças evitáveis como as arboviroses e internações, por meio das atribuições dos profissionais, uma vez que a articulação dos olhares dos diferentes trabalhadores da ESF possibilita o desenvolvimento de ações que ultrapassam a assistência curativa. $\mathrm{O}$ atendimento da população pelas equipes de saúde permite o desenvolvimento do trabalho de prevenção e tratamento contra o acometimento das doenças, antes da necessidade de internação do paciente (MAFRA, 2010), uma vez que as ações e práticas são estruturadas no coletivo e amplia o objeto de intervenção para além do âmbito individual e clínico. Essa peculiaridade requer mudanças na forma de atuação e organização do trabalho, e demanda diverso saberes (SILVA et al., 2005). A complexidade do processo de trabalho das ESF e contato cotidiano com a população exigem um processo de educação continuada. Como fator limitante do estudo foi identificado à falta de preenchimento dos questionários por algumas equipes das unidades de saúde.

\section{CONCLUSÕES}

Conclui-se que as doenças infectoparasitárias são consideradas problemas de saúde ambiental nos territórios das Equipes de Saúde da Família, mas as equipes apresentaram dificuldades em responder os questionários, mostrando possível falta de conhecimento sobre o tema, fato que pode influenciar na saúde da população residente nos territórios estudados. Portanto, realização de ações educativas continuadas e capacitações com as equipes sobre doenças infectoparasitárias, formas de contágio, veículos de transmissão, 
prevenção, tratamento tornam-se necessários para melhorar a percepção dos profissionais de saúde sobre os problemas ambientais recorrentes na área de abrangência de cada equipe para aperfeiçoar os atendimentos sobre doenças transmitidas por desordem ambiental.

\section{REFERÊNCIAS}

ALCÂNTARA, A. J. O.; PIERANGELI, M. A. P.. Composição gravimétrica dos resíduos sólidos urbanos e caracterização química do solo da área de disposição final do município de Cáceres/MT. In: SANTOS, J. E.; GALBIATI, C.; MOSCHINI, L. E.. Gestão e Educação Ambiental, água, biodiversidade e cultura. São Carlos: Rima, 2010. p.1-30.

ANDREAZZI, M. A. R.; BARCELLOS, C.; HACON, S.. Velhos indicadores para novos problemas : a relação entre saneamento e saúde. Rev. Panam Salud Publica, v.22, n.2, p.211-217, 2007.

BARBOSA, M. S. M.; LIMA, K. S. C.; FRIEDE, R. R.; MIRANDA, M. G.. A relação entre pobreza e degradação ambiental no Brasil sob a ótica dos indicadores PIB/IDH. Semioses, v.9, n.1, p.17-35, 2015. DOI: http://doi.org/10.15202/1981996X.2015v9n1p17

BRASIL. Lei n.12.305: Política nacional de resíduos sólidos. Brasília: Câmara dos Deputados, 2012.

BÜHLER, B. F.. Qualidade da água e aspectos sedimentares da bacia hidrográfica do rio Paraguai no trecho situado entre a Baía do late e a região do Sadao, município de Cáceres (MT), sob os enfoques quantitativos e perceptivos. Dissertação (Mestrado em Ciências Ambientais) Universidade do Estado de Mato Grosso, 2011.

BÜHLER, B. F.; SOUZA, C. A.. Aspectos sedimentares do rio Paraguai no perímetro urbano de Cáceres/MT. Geociências, São Paulo, v.31, n.3, p.339-349, 2012.

CAIRNCROSS, S.; FEACHEM R.. Environmental health engineering in the tropics: an introductory text. $2 \mathrm{ed}$. Chichster: Wiley \& Sons, 1993.

EJEMOT, R. I.; EHIRI, J. E.; MEREMIKWU, M. M.; CRITCHLEY J. A.. Hand washing for preventing diarrhoea. Cochrane Database Syst Rev, n.2, p.1-42, 2012.

FERREIRA, A. R.; JUNIOR, M. C.; SOUZA M. M. F.. Análise dos impactos ambientais na bacia hidrográfica do rio Paraguai/ Cáceres/MT: risco ecológico para o Pantanal Matogrossense. Os Desafios da Geografia Física na Fronteira do Conhecimento, v.1, 2017. DOI: http://doi.org/10.20396/sbgfa.v1i2017.1809

IBGE. Cidades 2015. Rio de Janeiro: Instituto Brasileiro de Geografia e Estatística. 2015.

LIMA, D. J. J.. Ambiente e saúde na cidade de Manaus: percepção de moradores (estudantes do ensino médio) sobre degradação ambiental e doenças infectoparasitárias. Tese (Doutorado em Geografia Física) - Universidade de São Paulo, São Paulo, 2013.
MAFRA, F.. 0 impacto da atenção básica em saúde em indicadores de internação hospitalar no Brasil. Brasília: Universidade de Brasília; 2010.

MINISTÉRIO DA SAÚDE. Datasus: Epidemiológicas e Morbidade. 2016.

OPS. Organizacion Panamericana de La Salud. Agua y saneamiento: evidencias para políticas públicas com enfoque em derechos humanos y resultados en salud pública. Washington: Organizacion Panamericana de La Salud, 2011.

PAIVA, R. F. P. S.; SOUZA, M. F. P.. Associação entre condições socioeconômicas, sanitárias e de atenção básica e a morbidade hospitalar por doenças de veiculação hídrica no Brasil. Cad. Saúde Pública, v.34, n.1, 2018.

PIGNATTI, M. G.. Saúde e ambiente: as doenças emergentes no Brasil. Ambient Soc., v.7, n.1, p.133-147, 2004.

PIUVEZAM, G.; FREITAS, M. R.; COSTA, J .V.; FREITAS, P. A . CARDOSO, P. M. O.. Fatores associados ao custo das internações hospitalares por doenças infecciosas em idosos em hospital de referência na cidade do Natal, Rio Grande do Norte. Cad. Saúde Colet., v.23, n.1, p.63-68, 2015.

PONTES, R. J. S.; RAMOS JR., A. N.; KERR, L. R. S.; BOSI, M. L. M.. Transição Demográfica e Epidemiológica. In: PONTES, R. J. S.; RAMOS JR., A. N.; KERR, L. R. S.; BOSI, M. L. M.; MEDRONHO, R. A.. Epidemiologia. 2 ed. São Paulo: ATHENEU, 2009. p.123-151.

PRUSS-USTUN, A.; BONJOUR, S.; CORVALÁN, C.. The impact of the environment on health by country: a meta-synthesis. Environ Health, v.7, n.7, p.1-10, 2008 a.

PRUSS-USTUN, A.; BOS, R.; GORE, F.; BARTRAM, J.. Safer water, better health: costs, benefits and sustainability of interventions to protect and promote health. Geneva: World Health Organization, 2008b.

PRUSS-USTUN, A.; CORVALÁN, C.. Preventing disease through healthy environments: towards an estimate of the environmental burden of disease. Geneva: World Health Organization, 2008c.

RHEINGANS, R.; DREIBELBIS, R.; FREEMAN, M. C.. Beyond the Millennium Development Goals: public health challenges in water and sanitation. Glob Public Health, v.1, n.1, p.31-48, 2006. DOI: http://doi.org/10.1080/17441690500443139

RIBEIRO, J. W.; ROOKE, J. M. S.. Saneamento básico e sua relação com o meio ambiente e a saúde pública. Dissertação (Especialização em análise ambiental) Universidade Federal de Juiz de Fora, 2010. 
SCHRAMM, J. M. A.; OLIVEIRA, A. F.; LEITE, I. C.; VALENTE, J. G.; GADELHA, A. M. J.; PORTELA, M. C.; CAMPOS, M. R. Transição epidemiológica e o estudo da carga de doenças no Brasil. Ciênc. Saúde Coletiva, v.9, n.4, p.897-908, 2004.

SILVA, I. Z. Q. J.; TRAD, L. A. B.. Team work in the PSF: investigating the technical articulation and interaction among professionals. Interface, Comunic., Saúde e Educ., v.9, n.16, p.25-38, 2005.

SILVA, R. V.; SOUZA, C. A.. Ocupação e degradação na margem do Rio Paraguai em Cáceres, Mato Grosso. Revista Brasileira de Gestão e Desenvolvimento Regional. Taubaté, v.8, n.1, p.125-152, 2012.
SOUZA, C. M. N.; MORAES, L. R. S.; BERNARDES, R. S. Classificação Ambiental e Modelo Causal de Doenças Relacionadas à Drenagem Urbana. In: Federación Mexicana de Ingeniería Sanitaria y Ciencias Ambientales; AIDIS Gestión inteligente de los recursos naturales: desarrollo y salud. Anais. 2002. p.1-7.

TEIXEIRA, J. C.; OLIVEIRA, G. S.; VIALI, A. M.; MUNIZ, S. S.. Estudo do impacto das deficiências de saneamento básico sobre a saúde pública no Brasil no período de 2001 a 2009. Eng. Sanit. Ambient, v.19, n.1, p.87-96, 2014.

A CBPC - Companhia Brasileira de Produção Científica (CNPJ: 11.221.422/0001-03) detém os direitos materiais desta publicação. Os direitos referem-se à publicação do trabalho em qualquer parte do mundo, incluindo os direitos às renovações, expansões e disseminações da contribuição, bem como outros direitos subsidiários. Todos os trabalhos publicados eletronicamente poderão posteriormente ser publicados em coletâneas impressas sob coordenação da Sustenere Publishing, da Companhia Brasileira de Produção Científica e seus parceiros autorizados. Os (as) autores (as) preservam os direitos autorais, mas não têm permissão para a publicação da contribuição em outro meio, impresso ou digital, em português ou em tradução. 